NASA Contractor Report 175028

AIAA-86-0450

\title{
Prediction of the Structure of Fuel Sprays in Gas Turbine Combustors
}

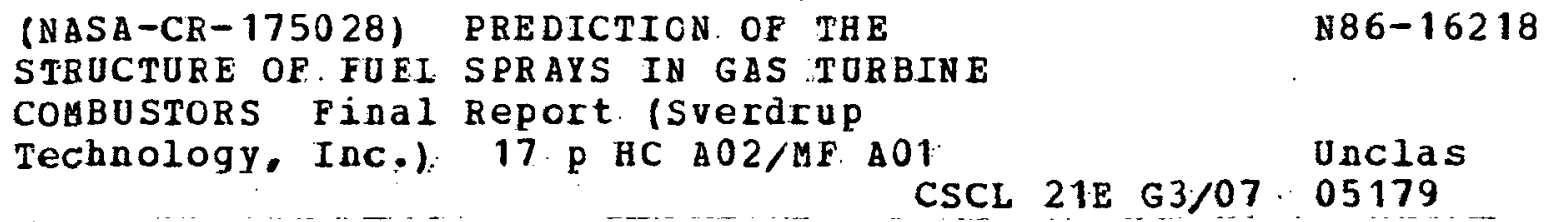

Jian-Shun Shuen

Sverdrup Technology, Inc.

Lewis Research Center

Cleveland, Ohio

December 1985

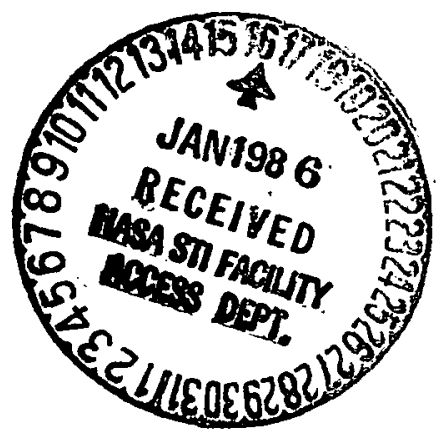

Prepared for the

Lewis Research Center

Under Contract NAS 3-24105 


\author{
Jian-Shun Shuen* \\ Sverdrup Technology, Inc. \\ Lewis Research Center \\ Cleveland, Ohio 44135
}

\section{Adstract}

The structure of fuel sprays in a combustion chamber is tneoretically investigated using computer models of current interest. Three representative spray models are considered: (1) a locally nomogeneous flow (LHF) model, which assumes infinitely fast interphase transport rates; (2) a deterministic separated flow (DSF) model, which considers finite rates of interphase transport but ignores effects of droplet/ turoulence interactions; and (3) a stochastic separated flow (SSF) model, which considers droplet/turbulence interactions using random sampling for turbulence properties in conjunction with random-walk computations for droplet motion and transport. Two flow conditions, i.e., swirl number $=0.72$ and 1.98 , are studied to investigate the influence of swirl on droplet life histories and the effects of droplet/turbulence interactions on flow properties. Comparison of computed results with the experimental data show that general features of the flow structure can be predicted with reasonable accuracy using the two separated-flow models. In contrast, the LHF model overpredicts the rate of development of the flow. While the SSF model provides better agreement with measurements than the DSF model, definitive evaluation of the significance of droplet/ curoulence interaction is not achieved due to uncertainties in the spray initial conditions.

\section{Nomenclature}

B mass transfer driving potential, Eq. (14)

$C_{D}$ drag coefficient

dp droplet diameter

D binary diffusivity

$f$ mixture fraction

$g$ mean square of mixture fraction fluctuations

$h$ heat transfer coefficient

$n_{f g}$ heat of vaporization

$k$ turbulence kinetic energy

Le Lewis number

Mp droplet mass

$\dot{m}^{\prime \prime}$ mass flux at droplet surface

$\dot{n}_{i}$ number flow rate of droplets in group $i$

$\mathrm{N}_{\mathrm{P}}, \mathrm{N}_{\mathrm{S}}$ convection correction, Eq. (15)

$P(f)$ probability density function (POF) of $f$

Pr Prandt 1 number

$r$ radial distance

$r_{0}$ combustion chamber radius

Re droplet Reynolds number

$S$ swirl number

Sc Scnmidt number

$S_{\phi}$ source term

$S_{p \phi}^{\phi} \quad$ droplet source term

time

temperature

axial velocity

radial velocity

*Senior Research Engineer.
W tangential velocity

$V_{j}$ volume of computational cell $j$

$x$ axial distance

$Y_{F}$ mass fraction of fuel vapor

$\Gamma$ exchange coefficient

$E$ rate of dissipation of turbulence kinetic energy

tnermal conductivity

viscosity

density

generic property

scalar property

\section{Subscripts}

p droplet property

F fuel vapor

s droplet surface property

$\infty \quad$ ambient property

\section{Superscripts}

(-) time-averaged quantity

( ) Favre-averaged quantity

\section{Introduction}

The objective of this investigation is to evaluate liquid fuel-spray combustion models for gas turbine combustors and furnaces. Numerous, spray models have Deen proposed and some are currencly being used in industry to assist gas turbine combustor development. 1,2 Recent reviews Dy Crowe, ${ }^{3}$ Law, ${ }^{4}$ Faeth $^{5}$ and Sirignano 6 aiscuss important aspects of spray modeling that are currently being addressed. Recent spray models differ in details, but generally may be divided into two categories: the locally homogeneous flow (LHF) inodels and separated flow (SF) models.

LHF models represent the simplest treatment of a multiphase flow and have been widely used to analyze sprays.7,8 The key assumption of the LHF model is that interphase transport rates are fast in comparison to the rate of development of the flow as a whole. This implies that all phases have the same velocity and temperature, and phase equilibrium is maintained at each point in the flow. Clearly, LHF models are only formally correct for flows containing infinitely small droplets. The potential range of application, however, can be much larger, since LHF models can be used when particle relaxation times are small in comparison to the characteristic time scales of the continuous phase. 5 LHF models have been extensively evaluated by Faeth and coworker s 5,7 and by Khalil and Whitelaw. It was found that LHF models provide a reasonable description of the structure of a spray, however, they generally overestimate the rate of development of the flow.

SF models nave been proposed to consider interpnase transport phenomena. Numerous separated-flow models have been proposed, Gosman 
and Jonns ${ }^{9}$ have divided them into three groups: discrete aroplet models, continuous droplet models and continuum formulation models. The discrete aroplet model was first proposed by Crowe 10 and later used by Gosman and Ioannides 11 and EI Bannawy and Whitelaw. 12 In this model droplets are divided into representative groups whose trajectories are tracked by solving the Lagrangian orainary-differential equations that govern droplet transport. The gas-phase conservation equations, with source terms included to represent the effect of the oroplets on the gas-phase, are solved in Eulerian coordinates. In the continuous droplet model proposed by Williams 13 and used oy westurook, 14 the droplets are represented by a statistical distribution function in a multidimensional space of droplet size, velocity, location and time. The properties of droplets are determined by solving the conservation equation of the distribution function, similar to the analys is of molecular properties in the kinetic theory of gases. In the continuum formulation models, droplets are treated as a continuous fluid, so that the gas and droplets are represented as interpenetrating continua. This formulation requires one droplet fluid phase for each droplet size. It is very expensive in terms of computer storage and time for practical sprays, since they generally have a wide range of droplet sizes. In most recent spray models the discrete droplet formulation has been adopted, since it reduces numerical diffusion and computer storage requirements while providing a convenient framework for dealing with multiple droplet size and complex interpnase transport phenomena. The present investigation emphasizes the discrete droplet mode 1 .

Many aiscrete droplet models neglect the effects of turbulence on interphase transport.1,2 - This implies that droplets follow deterininistic trajectories, yielding the aeterministic separated flow (USF) model. Neglecting the effects of turDulence on droplet transport is appropriate when characteristic oroplet relaxation times are large in comparison to characteristic times of turbulent fluctuations.:- Few practical sprays however, satisfy this condition. Dukowicz, 15 Gosman and Ioannioes 11 and Faeth and coworkers 16-20 have adopted stochastic metnods to treat the turbulencel droplet interactions. In the stochastic separated flow (SSF) model, droplets are assumed to interact with turbulent eddies whose properties are-obtained by randoin sampling. An appropriate number of random samples is taken for each droplet group so that statistically significant mean droplet properties can be obtained. The iormulation of the SSF model was first proposed by Gosman and Ioannides 11 to study droplet dispersion. Faeth and coworkers 16-20 furtner developed the model to include the effects of turbulence on interphase neat and mass transport. The SSF model has been extensively evaluated by Faeth and coworkers $16-20$ in a wide variety of parabolic flows and the results have been very encouraging.

The present investigation extends the SSF models of Refs. 16 to 20 to consider swirling and recirculating flows. Results from LHF and DSF models are also presented in order to hignlight the effects of finite interphase transport rates and droplet/turbulence interactions.

\section{Theoretical Methods}

The LHF model is equivalent to the analysis of a variable density single-phase flow. The effects of the dispersed phase appear in the upstream boundary conaitions and the representation of state relationships. The present LHF model is very similar to that reported in earlier
work on evaporating ano combusting sprays. $19,20,22$

\section{Gas Pnase}

The major assumptions of the gas-phase formulation are as follows: (1) the flow is steady, axisymmetric, and swirling, (2) exchange coefficients of ali species and thermal energy are the same, (3) kinetic energy of the mean flow is negligible, (4) combustion is diffusion controlled, (5) radiative neat transfer is neglected, and (6) the sprays are dilute so that droplet collisions and the displacement of the gas by the droplets can be neglected. Favre-averaged governing equations are solved. The conserved-scalar formulism is used to obtain scalar properties. Turbulence closure. is achieyed by employing a $k-\varepsilon-\mathrm{g}$ turbulence model.12,21

The governing equations of the gas-phase can be written in the general form, 12

$$
\begin{aligned}
\frac{\partial}{\partial x}(\bar{\rho} \tilde{u})+\frac{1}{r} \frac{\partial}{\partial r}(\tilde{r} \tilde{\rho v \phi})=\frac{\partial}{\partial x}\left[\Gamma \frac{\partial \tilde{\phi}}{\partial x}\right] \\
+\frac{1}{r} \frac{\partial}{\partial r}\left[r r \frac{\partial \tilde{\phi}}{\partial r}\right]+S+S_{p \phi}
\end{aligned}
$$

where $\tilde{\phi}=\bar{\rho} \phi / \bar{\rho}$ is tine Favre-averaged dependent variable. The expressions for the exchange coefficient $\Gamma$ and the source term $S$ can be found in Refs. 12 and 23 . The term ' $S_{p_{\phi}}$ is used for tine $S F$ models and represents the interaction between tine droplets and the gas phase and is obtained from the droplet trajectory calculations, as will be described later. Because of the dilute spray assumption the influence of droplets on turbulence properties is neglected.

A gaseous diffusion flame structure is assumed for the combustion analys is and the droplets are treated as distributed sources of fuel vapor. The presence of envelope flames around or wake flames benind individual droplets is neglected since the majority of droplets spend most of their lifetimes in fuel rich regions. Experimental confirmation of this assumption can De found in Refs. 5 and 24.

The Favre-averaged scalar properties (e.g., temperature and species concentrations) are calculated from the probability density function (PUF) of mixture fraction, $\widetilde{P}(f)$, and state relationships of scalar properties as a function of mixture fraction, $\psi(f)$,

$$
\tilde{\psi}=\int_{0}^{1} \psi(f) \tilde{P}(f) d f
$$

wnile $\bar{\rho}$ is found from

$$
\bar{\rho}=\left(\int_{0}^{1} \frac{1}{\rho(f)} \tilde{p}(f) d f\right)^{-1}
$$


- Elippeo baussian function is assumed for $\tilde{p}_{i}(1)$ ano the most probable value and variance are determined $b y$ i and $g$. State relationships are ovtainea by tinermodynamic equilibrium calcuiations using tne LEC-70 computer progran developed oy cordon and Mcoriae.25 The SSF mode? requires tine time-averaged $P O F, \bar{P}(f)$, whicn can oe found as follows:

$$
\bar{P}(f)=\bar{\rho} / 0(f) \bar{P}(f)
$$

Jroplet Pnase

T:le major assumptions involved in the droplet iransport calculations have been discussed in detail by $L_{a w}{ }^{C}$ and $F$ aetin ${ }^{5}$ and will not be

repeateo nere. In the present investigation, the inin-skin approximation is useo for droplet evaporation. This implies that the bulk mass of a uropiet remains at its initial condition and only ine surface of the aroplet is heated during evaporation. This approximation is convenient for the conserved scalar approach of the gas field since energy aosoroed by the liquid is neglected. T:le justification for this approximation is discussed in Refs. 5 and 20.

Droplet irajectories are solved in Lagrangian coorginates. The governing equations of droplet iranspori are given delow.

uroplet inotion. The position, axiai, radial ano iangential momentum equations are

$$
\begin{gathered}
\frac{d \vec{u}_{p}}{a t}=\vec{u}_{p} \\
\frac{a u_{p}}{d t}=\frac{3}{4} \frac{\partial C_{D}}{\rho_{p} d_{p}}\left(u-u_{p}\right)\left|\vec{u}-\vec{u}_{p}\right| \\
\frac{\partial v_{p}}{\partial t}=\frac{3}{4} \frac{\partial C_{D}}{\rho_{p} d_{p}}\left(v-v_{p}\right)\left|\vec{u}-\vec{u}_{p}\right|+\frac{w_{p}^{2}}{r} \\
\frac{\partial w_{p}}{\partial t}=\frac{3}{4} \frac{\rho C_{D}}{\rho_{p} d_{p}}\left(w-w_{p}\right)\left|\vec{u}-\vec{u}_{p}\right|-\frac{v_{p} w_{p}}{r}
\end{gathered}
$$

The standaro drag coefficient is approximated as

follows:

$$
\begin{aligned}
L_{J} & =\frac{24}{\operatorname{Re}}\left(1+\frac{R e^{2 / 3}}{6}\right), & & \operatorname{Re} \leq 1000 \\
& =0.44 & & \operatorname{Re}>1000
\end{aligned}
$$

where the aroplet Reynolds number, Re, is defined as $\operatorname{Re}=\rho\left|\vec{u}-\vec{u}_{p}\right| d_{p} / u$.

Droplet evaporation. The droplet heat and mass transport equations are as follows: 26

$$
\frac{d d p}{d t}=-\frac{2 n^{\prime \prime}}{d_{p}}
$$

$$
\begin{gathered}
\frac{m^{\prime \prime} d_{p}}{\rho D}=2 N_{s} \ln (1+B) \\
T_{p}=T-\frac{m^{\prime \prime} h_{f g}}{h}
\end{gathered}
$$

$$
\begin{gathered}
\frac{h_{p}}{\lambda}=\frac{2 N_{p} \ln (1+B)^{L e^{-1}}}{\left[(1+B)^{\left.L e^{-1}-1\right]}\right.} \\
B=\frac{\left(Y_{F s}-Y_{F_{\infty}}\right)}{\left(1-Y_{F s}\right)} \\
N_{p} \text { or } N_{S}=1+\frac{0.276 \operatorname{Re}^{1 / 2}(\operatorname{Pr} \text { or } S c)^{1 / 3}}{L 1+1.232 /\left(\operatorname{Re}(\operatorname{Pr} \text { or SC })^{4 / 3 j} 1 / 2\right.}
\end{gathered}
$$

The fuel vapor concentration and temperature at the liquid surface are related through the fuel vapor pressure characteristics

$$
Y_{F S}=f\left(T_{S}, p\right)
$$

Time-averaged gas properties and instantaneous eddy properties should be used for droplet trajectory calculations in the DSF and SSF models, respectively. Time-averaged scalar properties $c$ an be found directly from the conserved-scalar formulation. 27 However, to convert Favreaveraged velocities to time-averaged velocities requires correlations of velocity-density fluctuations. To avoid undue complications in the analysis, the Favre-averaged gas velocities are used in the calculations. Jeng and Faeth ${ }^{28}$ estimated the difference betwicen the two averaged velocities in their diffusion flame to be less than 5 percent.

\section{Droplet/Turbulence Interaction}

In the SSF model, the effects of turbulence on droplet transport are treated by a stochastic approach. The complete formulation of the stochastic method can be found in Refs. 9 and 16 to 20 anc unly a brief description is given here.

Lagrangian equations of droplet motion and transport are solved by dividing the droplets into $n$ groups (defined by initial position, size and velocity) at the nozzle exit and computing their life nistories in the flow field. The droplets are assumed to interact with a random distribution of eddies along their trajectories. The eady properties are obtained by random sampling of the POF's of velocity and mixture fraction. To simplify the analysis, velocity and mixture fraction are assumed to be statistically independent. The turbulence is assumed to be isotropic and to have a Gaussian PDF in the velocity fluctuations. Although the time-averaged PDF should strictly be used, the Favre-averaged PDF is used here for the same reason as in the DSF analysis.

The scalar eddy properties are obtained by sampling the time-averaged $P D F, \bar{P}(f)$, for an 
instantaneous value of $f$ and then obtaining the corresponding scalar properties from the state relationsnips. The droplet/eddy interaction time is assumed to be the minimum of either the eddy lifetime or the transit time for the droplet to cross the eddy. Details of the specification of these times and their calibration can be found in Refs. 10 to 20 .

\section{Droplet Source Terms}

The influence of droplets on the turbulence is neglected in this study and no source terms from the droplets are included in the $k, \varepsilon$ and $g$ equations. The droplet mass and momentum source terms appearing in Eq. (1) are computed after the oroplet transport equations are integrated.

The mass and axial momentum source terms for each computational cell $\mathrm{j}$ are calculated from the net change of droplet mass and axial momentum for each droplet group traversing tnis cell, as follows:

$$
\begin{array}{r}
s_{p m j}=v_{j}^{-1}\left(\sum_{i=1}^{n} \dot{n}_{i}\left(m_{p i}-m_{p i}\right)\right)_{j} \\
s_{p u j}=v_{j}^{-1}\left(\sum _ { i = 1 } ^ { n } n _ { i } \left(\left(m_{p i} u_{p i}\right)_{i n}\right.\right. \\
\left.\left.-\left(m_{p i} u_{p i}\right)_{\text {out }}\right)\right)_{j}
\end{array}
$$

The droplet radial and tangential momentum source terms, however, cannot be calculated in this manner aue to the additional force terms in the respective droplet equations of motion (cf. Eqs. (7) and (8)). These source terms are obtained by directly computing the change of radial and tangential momentum due to drag, as follows:

$$
\begin{array}{r}
s_{p v j}=v_{j}^{-1} \int_{t_{i n}}^{t}\left(\sum_{i=1}^{n} \frac{3}{4} n_{i} m_{p i} \frac{\rho^{d}}{\rho_{p} d_{p}} c_{D}\right. \\
\times\left(v_{p i}-v-\left|\vec{u}_{p i}-\vec{u}\right|\right) d t \\
s_{p w j}=v_{j}^{-1} \int_{t_{i n}}^{t_{o u t}}\left(\sum_{i=1}^{n} \frac{3}{4} \dot{n}_{i} m_{p i} \frac{\rho}{\rho_{p} d_{p}} c_{D}\right. \\
\left.\times\left(w_{p i}-w\right)\left|\vec{u}_{p i}-\vec{u}\right|\right) d t
\end{array}
$$

Boundary and Initial Conditions

The elliptic nature of the gas-phase governing equations requires that boundary conditions be specified along all surfaces enclosing the computational domain. Measured values of mean axial velocity and temperature are prescribed at the inlet. Inlet radial velocity is taken as zero and the tangential velocity is specified to have a solid-body rotation profile. Second derivatives of all dependent variables are assumed to be zero at the exit. Axisymetry is assumed and adiabatic and non-slip conditions are specified along all solid surfaces.

The Lagrangian formulation of the droplet transport equations requires the specification of initial conditions for the droplets to start the integration. The initial conditions required in the present analysis include oroplet velocities, location, and size distribution. This information, nowever, is rarely available in practical flows. As a result, approximations of initial conoitions are used in the predictions. Initial aroplet locations and velocities are estimated from the limited available experimental information. The droplet size is assumed to have a Rosin-Rammler size distribution. Upon impingement on a wall, the droplets are assumed to bounce Dack elastically with an angle of reflection equal to the angle of incidence.

\section{Numerical Solution Procedure}

A modified version of the TEACH computer program is used to solve the gas-phase governing equations. It includes an improved finitedifference procedure, the Bounded Skew-Upwind Differencing (BSUD) method developed by Raithby. 30 The pressure field is estimated using the Pressure Implicit Split Operator (PISQ) predictor-corrector technique developed by Issa. 31 The axisymmetric flow domain is divided into relatively fine meshes, i.e., 60 by 60 . For the separated-flow analysis, the ordinary differential equations governing tine motion and transport of each oroplet group are integrated using a fourth-order RungeKutta method. The numerical integration of droplet trajectories is started from the fuel nozzle exit where the initial conaitions of the droplets are prescribed. Seven hundred and 2800 trajeccories are computed for the DSF and SSF models, respectiveiy, in all cases. The solution procedure follows the PSI-CELL (Particle-source in (ell) iterative metnod of crowe et al.10 The gas-pnase solution is odtained with droplet source terins from the previous iteration, then the droplet trajectories and the new mass and momentum source terms in eacn cell are calculated. The iteration cycle is repeated until the solutions for both phases converge. Generally, convergence is achieved in 1200 interations for the separatedflow calculation and 750 iterations for the LHF calculation. The CPU time requirements are approximately 600,1400 , and $2800 \mathrm{sec}$, respectively, for tne LHF, DSF, and SSF models on the CRAY-1S/2200 computer.

\section{Results and Discussion}

The theoretical models described previously were applied to the liquid-fueled furnace used by Knalil et al.29 The geometry of the combustion cnamber is shown in Fig. 1. Two swirl numbers $(S=0.72$ and 1.98$)$ were considered and the fuelair equivalence ratio was fixed at 0.8 .

The predicted and measured temperature profiles at different axial locations are given in Fig. 2 for $S=0.72$. The results snow that both the DSF and SSF models underpredict the temperature 
in the near inlet region, while the LHF model overpredicts the temperature, especially at large radii $\left(r / r_{0}>0.8\right)$. The discrepancies between tne predictions of the two separated-flow models and the measurements are due to the underestimation of droplet spreading and evaporation, which is probably caused by uncertainties in the spray initial conditions. The SSF model snows better agreement than the DSF model because of better predictions of droplet evaporation and spreading rates. The overestimation of temperature by the LHF model is due to the assumption of infinitely fast interphase transport.

Far downstream of the combustor inlet, all three models predicted nigher temperatures than tne measurements. This is attributed to the neglect of radiative heat transfer. However, these errors in the predicted temperatures do not significantly affect the performance of the three models and hence, the conclusions of this study. both the experimental data and the separated-flow model predictions showed high temperature peaks near the combustion chamber wall at downstream locations. The peak temperatures occur in regions where droplets have traversed and evaporated. The LHF model did not predict a temperature peak near the chamber wall because this model does not consider discrete droplet motion.

Figure 3 presents the experimental data and predictions for $S=1.98$. The temperature profiles are more uniform for $S=1.98$ than for $S=0.72$ (Figs. 2 and 3 ). This is due to the increased mixing obtained with increased swirl. The nigner swirl also produces a stronger recirculating flow (i.e., more fuel vapor is transported upstream) and hence higher temperatures are obtained in the upstream region $(x<0.24 \mathrm{~m})$ for $S=1.98$. The agreement between the predictions and the measurements is similar to the lower swir number case, except close to the inlet at large radii, where both the DSF and SSF models significant ly underestimate the temperature. Very few fuel droplet groups are predicted to arrive in these regions (i.e., close to the inlet at large radii). This is probably caused by inaccurate initial droplet conditions. The fuel vapor concentration in these regions is therefore underpredicted which results in much lower temperatures than the experimental data. For both swirl number cases in regions far downstream, the DSF mode 1 predicts much nigher temperature peaks, less uniform temperature distributions, and larger discrepancies with measurements than the SSF model (Figs. 2 and 3 ). This is because the DSF model neglects turbulent dispersion and hence, underestimates droplet spreading.

Radial profiles of gas-phase axial velocity are presented in Figs. 4 and 5 for $S=0.72$ and 1.98 , respectively. The boundaries of the central recirculation zones for $S=1.98,1.25$ and 0.94 are shown in Fig. 6. For clarity in presentation, the results of only the SSF model are given. It can be. seen that Doth the magnitude of the reverse velocities and the size of the recirculation zones are underpredicted, the errors increase with increasing swirl number. The implication of the underestimation of the recirculation can be seen in the temperature distributions in Fig. 3. The experimental data shows more uniform distributions Decause of greater mixing caused by larger reverse velocities. The poor performance of the model in predicting the size of the central recirculation zone and the reverse velocity may be partly attributed to the $k-\varepsilon$ model used in the present analysis. It is well known that $k-\varepsilon$ models have not been very successful in predicting highly swirling flows. 32 Beyond the central recirculation zone, the predicted axial velocities are nigher than the measured values due to the higher predicted temperatures.

The predicted and measured radial profiles of gas-phase tangential velocities for the higher swirl number case are illustrated in Fig. 7. The calculated tangential velocities are smaller than the measured values at almost all locations. This aiscrepancy is attributed to uncertainties in the inlet swirl velocities. It snould also be noted that the swirl numbers quoted by Knalil et al. 29 for the experimental data were estimated from the swirler vane angle rather than direct measurements and may introduce some error. The underestimation of the tangential velocities is partially responsible for the errors in the predictions of flow reversal.

The predicted axial profiles of liquid mass flow rate are shown in Fig. 8 for $S=0.72$ and 1.98. As expected, the LHF model produces unrealistically high evaporation rates. 19 This nigh rate of evaporation is due to the method used to obtain the inlet boundary conditions and the assumption of infinitely fast interphase transport rates. The results of the two separatedflow models appear reasonable, but lack of experimental data prevents quantitative comparisons. The SSF model predicts higher evaporation rates than tne DSF model Decause it considers the effects of turbulence on interphase transport, which ennances the rate of evaporation. 19,20
Tne influence of swirl strength on the overall evaporation rate is clearly indicated in Fig. 8, which shows faster evaporation for higher inlet swirl. Tnis can de explained by the interaction between the central recirculation zone and the droplet trajectories. In the high swirl case a 1 arge reverse flow is generated and most small droplets are trapped in the recirculation zone and evaporate there, resulting in intensive burning and high temperatures in this region. Large aroplets which penetrate the central recirculation zone burn further downstream or near the combustor wall. In the low swirl case, most droplets survive the central recirculation zone and continue to evaporate in the far downstream region. Since the mixing is weak outside the reverse flow region, high fuel vapor concentration along the droplet trajectories produce local high temperature peaks (see Fig. 2). In addition to the influence on droplet trajectories, the larger reverse flow zone recirculates more hot combustion products from downstream regions and increases the temperature at near inlet locations. This in turn contributes to a nigher evaporation rate for the hign swirl case.

The predicted axial profiles of droplet SMD are illustrated in Fig. 9 for $S=0.72$. The values presented here were obtained by integrating over the cross-sectional area at each axial loca-

tion. Following Knalil et al., 29 the spray was assumed to have an initial SMD of $127 \mu \mathrm{m}$. A Rosin-Rammler size distribution, with 15 size 
groups and size range of 10 to $290 \mu \mathrm{m}$, was employed in the calculations. As the droplets move downstream, the SMO increases and then aecreases. The initial increase of SMD is caused Dy the reduction of the number of small droplets, due to the preferential evaporation of small droplets (i.e., small droplets evaporate faster than large droplets). When most of the small aroplets nave completely evaporated, the SMD begins to decrease because of the continuous evaporation of large aroplets. The DSF and SSF models produce similar results, but tine SSF model produces more rapia variations in the SMD than the DSF model because it predicts a nigher evaporation rate.

\section{Conclusions}

Current theoretical spray models have been used to predict properties of fuel sprays in a cylindrical combustion chamber. The results showed that general features of the gas-phase flow field can be predicted reasonably well. However, the evaluation of model performance for the dispersed phase is not conclusive mainly due to inadequate knowledge of the droplet initial conditions and a lack of experimental data for downstream droplet properties. Some quantitative differences between predictions and me "urements, such as size of the recirculation zone, magnitude of the tangential velocity, temperature distribution close to the inlet, could be relaced to uncertainties in the gas-phase upstream boundary conditions and also to droplet initial conditions. The need for a set of benchmark experimental data on combustor fuel sprays with characterization of inlet conditions and flow measurements in both pnases has long been recognized.3,11,12 The present investigation reemphasizes this need.

The LHF method yields unrealisticaliy nigh evaporation rates and overestimates the development of the flow field. Therefore, it has only limited utility in modeling practical sprays. The dispersion model gives less concentrated droplet trajectories and consequentially better temperature distributions than the deterministic metnod. Turbulence/droplet interaction enhances the rate of evaporation and produces higher fuel vapor concentration in the upstream portion of the combustor, where most droplets evaporate. Inclusion of these effects results in better predictions of the temperature, especially in. regions near the wall and close to the inlet. Although the SSF model appears to have certain advantages over DSF model, the differences between the two predictions are not large compared to the uncertainties in the droplet initial conditions. The lack of experimental data providing important inlet conditions and detailed flow measurements in both phases prevents a decisive evaluation of the two separated-flow models.

\section{Acknowledgement}

This research was sponsored by the National Aeronautics and Space Administration, Contract No. NAS 3-24105, under the technical management of Daniel L. Bulzan of the Lewis Research Center.

\section{References}

1. Swithenbank, J., Turan, A., and Felton, P.G., Gas Turbine Combustor Design Problems, Lefebrre, A.H. ed., pp. 249-314. Hemisphere Publishing, Wasningtu?, 1980.

2. Mongia, H.C. and Smitn, K. "An Empirical Analytical Design Methodology for Gas Turbine Combustors." AIAA Paper No. 78-988, 1978.

3. Crowe, C.T., "Review-Numerical Models for Dilute Gas-Particle Flows," J. Fluid Engrg., Vol. 104, 1982, pp. 297-503.

4. Law, C.K., "Recent Advances in Droplet Vaporization and Combustion," Prog. Energy Combust. Sc., Vol. 8, 1982, pp. 171-201.

5. Faeth, G.M., "Evaporation and Combustion of Sprays," Prog. Energy Combust. Sci., Vol. 9, 1983, pp. 1-76.

6. Sirignano, W.A., "Fuel Vaporization and Spray Combustion Theory," Prog. Energy Combust. Sci., Vol. 9, 1983, pp. 291-322.

7. Shearer, A.J., Tamura, H., and Faeth, G.M., "Evaluation of a Locally Homogeneous Flow Model of Spray Evaporation," J. of Energy, Vol. 3, 1979, pp. 271-278.

8. Knalil, E.E. and Whitelaw, J.H., "Aerodynamic and Thermodynamic Characteristics of Kerosene-Spray Flames, "Sixteenth Symposium (International) on Combustion, The Combustion Institute, Pittsburgn, 1977, pp. 569-576.

9. Gosman, A.D. and Johns, R.J.R., "Computer Analysis of Fuel-Air Mixing in DirectInjection Engines," SAE Paper No. 800091, 1980 .

10. Crowe, C.T., and Stock, D.E., "A Computer Solution for Two-Dimensional Fluid-Particle Flows," Int. J. Num. Method. Engr., Vol. 10, 1976, pp. 185-196.

11. Gosman, A.D. and Ioannides, E.,. "Aspects of Computer Simulation of Liquid-Fueled Combustors," AIAA Paper No. 81-0323, 1981.

12. El Banhawy, Y., and Whitelaw, J.M., "Calculation of the Flow Properties of a Confined Kerosene-Spray Flame," AIAA J., Vol. 18, 1980, pp. 1503-1510.

13. Williams, F.A., Combustion Theory, AddisionWesley, Reading, MA, 1965.

14. Westorook, C.K., "Three Dimensional Numerical Modeling of Liquid Fuel Sprays," Sixteenth Symposium (International) on Combustion, The Combustion Institute, Pittsburgh, 1977, pp. 1517-1526.

15. Dukowicz, J.K., "A Particle-Fluid Numerical Model for Liquid Sprays," J. Comp. Phys., vol. 35,1980 , pp. 229-253. 
16. Snuen, J.S., Solomon, A.S.P, Zhang, Q.F. and Faeth, G.M., "Structure of Particle-Laden Jets: Predictions and Measurements," AIAA J., Vol. 23, 1985, pp. 396-404.

17. Solomon, A.S.P., Shuen, J.S,, Zhang, Q.F., and Faeth, G.M., "Structure of Nonevaporating Sprays, Part I.: Initial Conditions and Mean Properties," AIAA J., Vol. 23, 1985, pp. $1548-1555$

18. Ibia., "Structure of Nonevaporating Sprays, Part II: Drop and Turbulence Properties," AIAA J., Vol. 23, 1985, pp. 1724-1730.

19. IDid., "Measurements and Predictions of the Structure of Evaporating Sprays, J. Heat Transfer, Vol. 107, 1985, pp. 679-686.

20. Shuen, J.S., Solomon, A.S.P., and Faeth, G.M., "Urop-Turbulence Interactions in a Diffusion Flame," AIAA J., in press.

21. Lockwood, F.C. and Naguib, A.S., "The Prediction of the Fluctuations in the Properties of Free, Round Jet, Turbulent, Uiffusion Flames," Comb. Flame, Vol. 24, 1975, pp. 109-124.

22. Mao, C.P., Szekely, G.A., Jr., and Faeth, G.M., "Evaluation of a Locally Homogeneous Flow Model of Spray Combustion," J. Energy, Vol. 3, 1980, pp. 79-87.

23. Gosman, A.U., Ioannides, E., Lever, D.A., and Cliffe, K.A., "A Comparison of Continuum and Discrete Droplet Finite-Difference Models Usea in the Calculation of Spray Combustion in Swirling Turbulent Flows, "AERE Harwell Report TP 865, 1980.

24. Onuma, Y. and Ogasawara, M., "Further Experiments on a Spray Combustion Flame," Sixteenth Symposium (International) on Combustion, The Combustion Institute, Pittsburgn, 1977, pp. 561-576.
25. Gordon, S. and McBride, B.J., "Computer Program for Calculation of Complex Chemical Equilibriun Compositions, Rocket Performance, Incident and Reflected Shock, and

- Cnapman-Jouget Detonations," NASA SP-273, 1976.

26. Faeth, G.M., "Current Status of Droplet and Liquid Combustion," Prog. Energy Combust. Sci., Vol. 3, 1977, pp. 191-224.

2i. Bilger, R.W., "Turbulent Jet Diffusion Flames," Prog. Energy Combust Sci., Vol. 1, 1976, pp. 87-109.

28. Jeng, S.M. and Faeth, G.M., "Species Concentrations and Turbulence Properties in Buoyant Methane Diffusion Flames," J. Heat Transfer, Vol. 106, 1984, pp. 721-727.

29. Knalil, K.H., El Mahallawy, and Moneib, H.A., "Effect of Combustion Air Swirl on the Flow Pattern in a Cylindrical 0il Fired Furnace," Sixteenth Symposium (International) on Combustion, The Combustion Institute, pittsdurgn, 1977, pp. 135-143.

30. Raithby, G.0., "Skew-Upstream Differencing Scneme for Problems Involving Fluid Flow," Computer Methods in Applied Mechanics and Engineering, VoT. 9, No. 2, 1976, pp. 153-164.

31. Issa, R.I., "Nurnerical Methods for Two- and Three-Uimensional Recirculation Flows," Computational Methods for Turbulent, Transonic, and Viscous Flows, Essers, J.A. ed., Hemisphere Publishing, New York, 1983, pp. $183-212$.

32. Hutchinson, P., Khali I, E.E., and Whitelaw, J.H., "The Measurements and Calculation of Furnace-Flow Properties," J. Energy, Vol. 1, 1977, p. 212. 


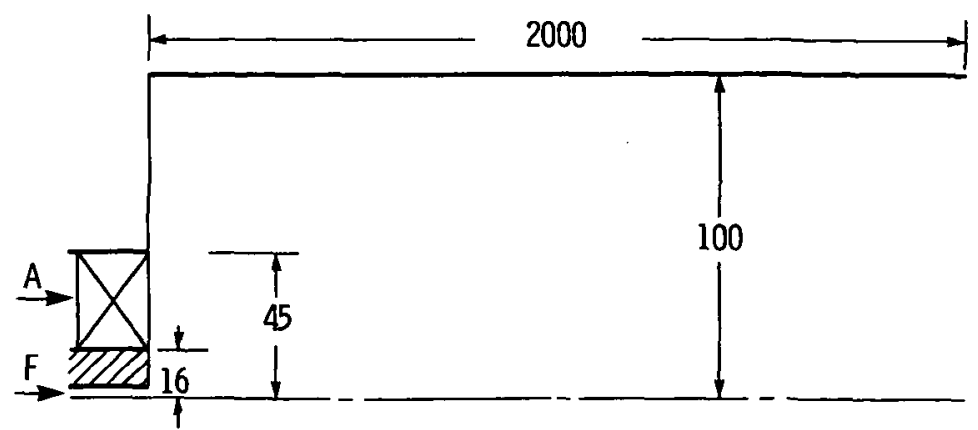

Figure 1. - Geometry of the combustion chamber. (All dimensions in $\mathrm{mm}$ ).
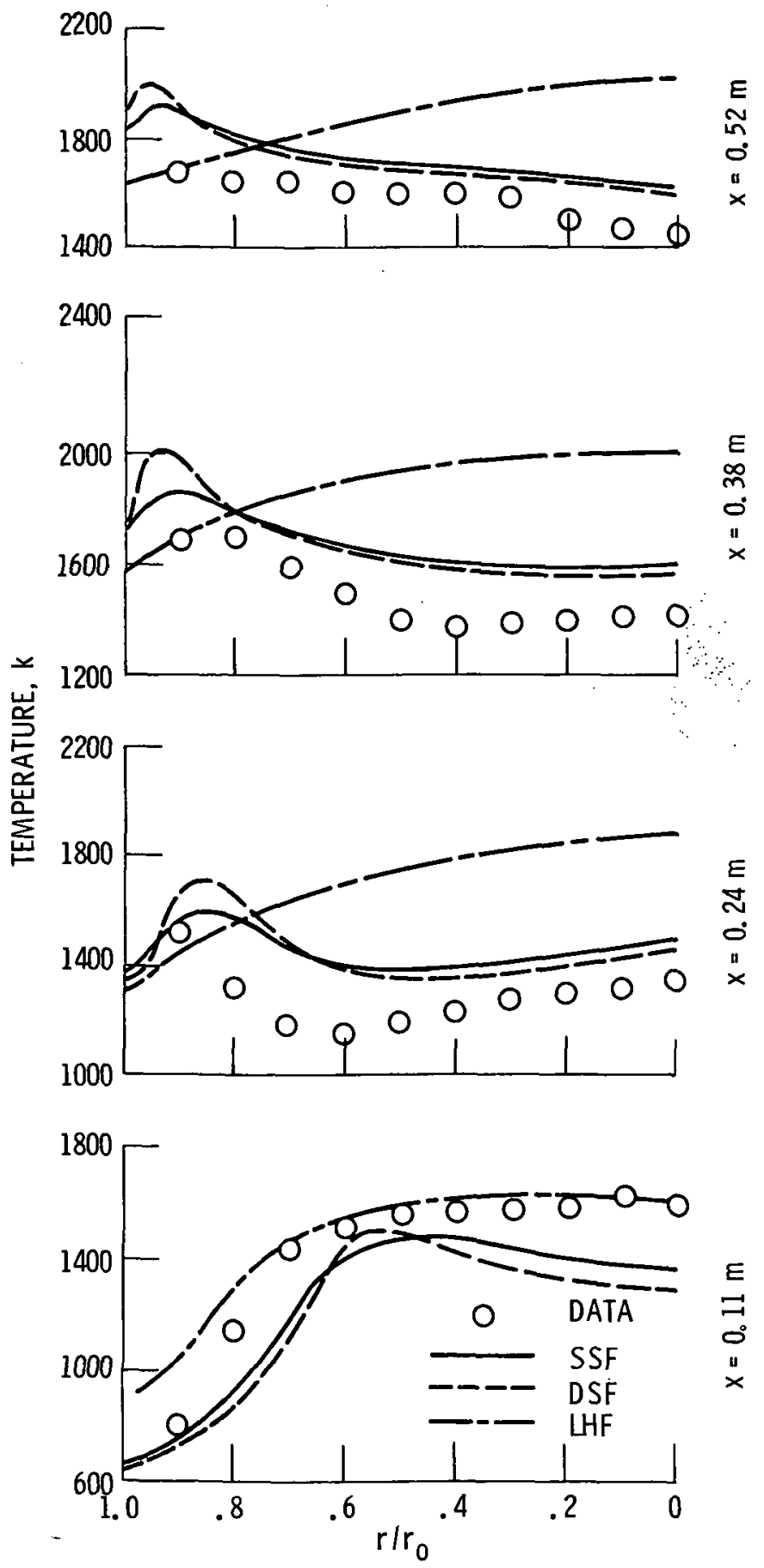

Figure 2. - Radial profiles of temperature, $S=0.72$. 

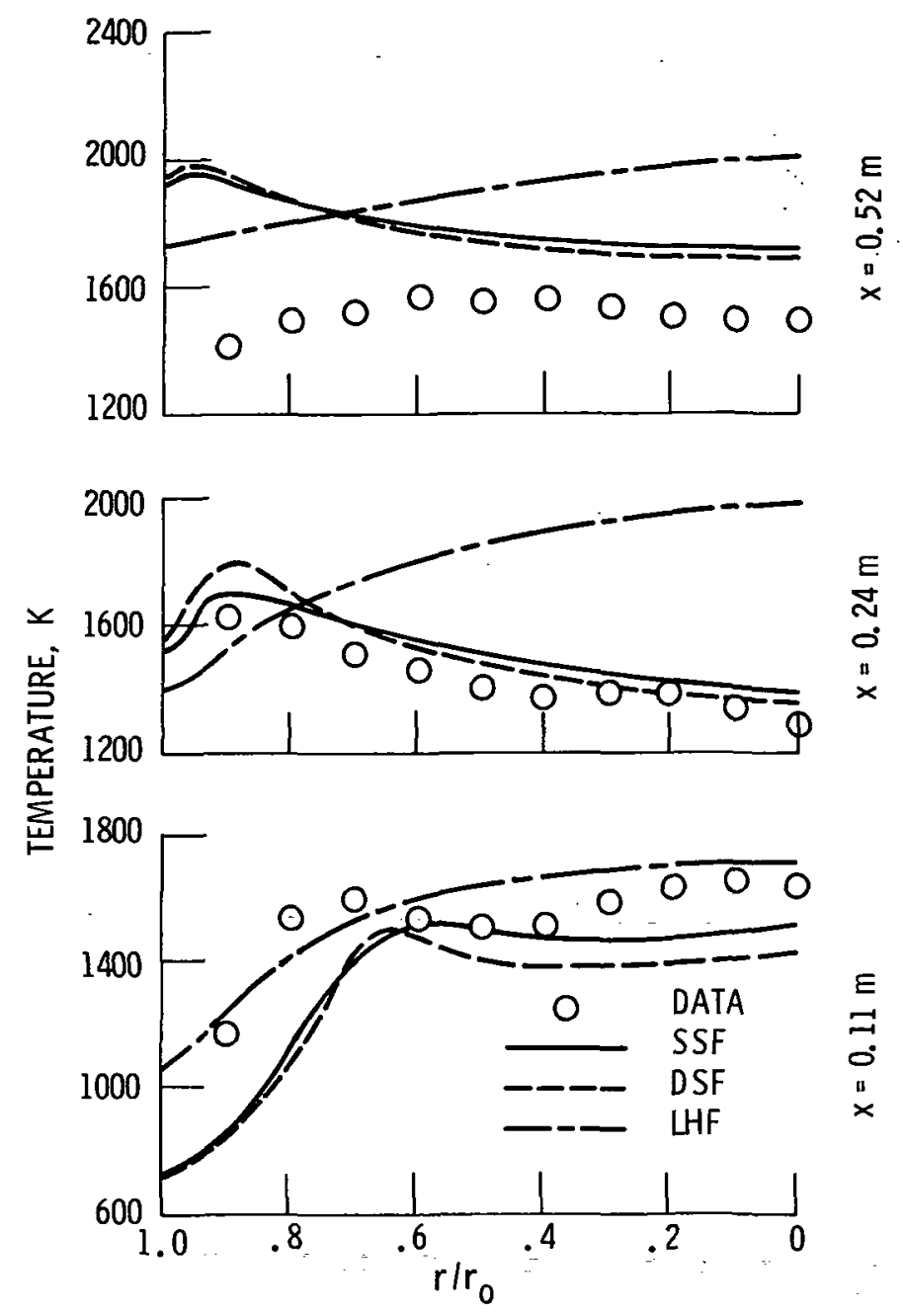

Figure 3. - Radial profiles of temperature, $S=1.98$. 

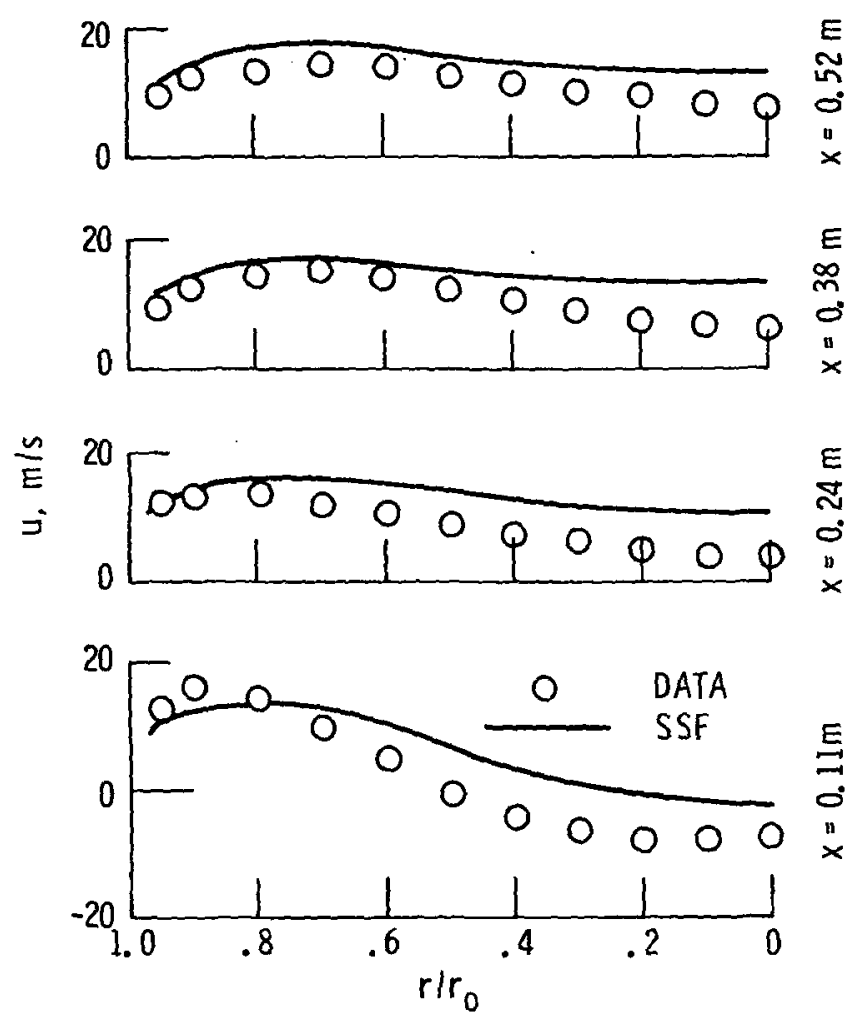

Figure 4. - Radial profiles of axial velocity, $S=0.72$. 

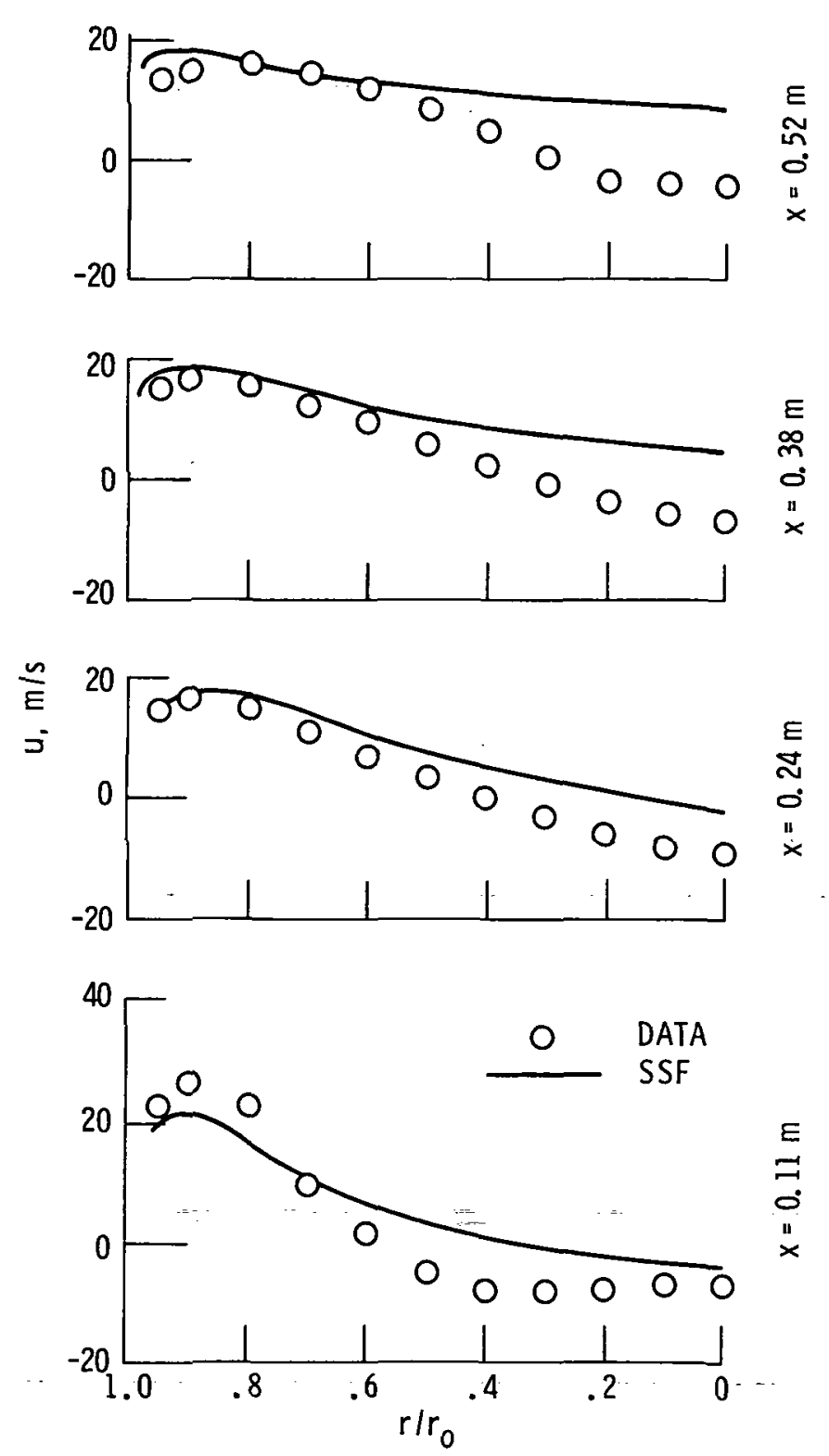

Figure 5. - Radial profiles of axial velocity, $S=1.98$. 

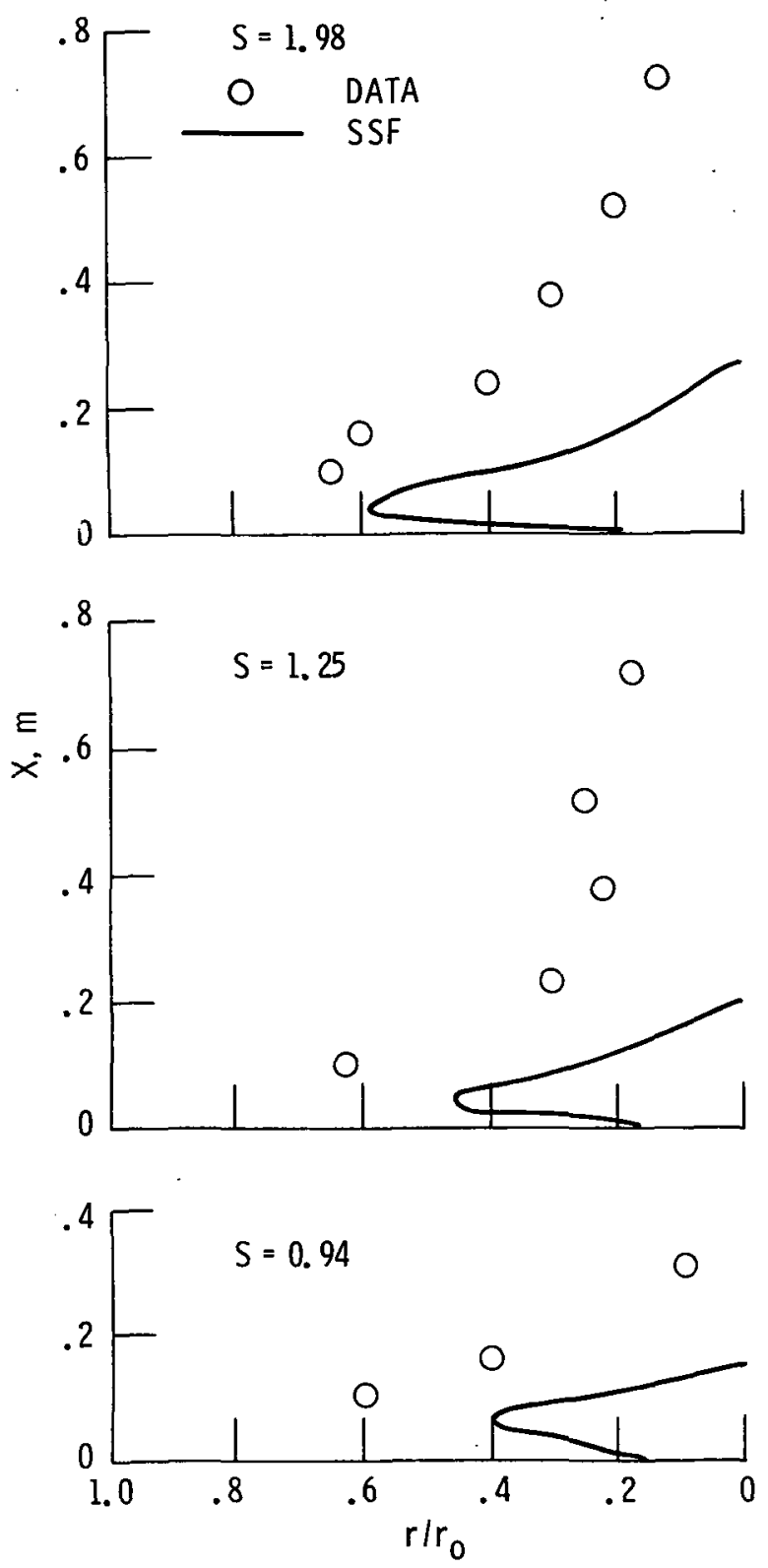

Figure 6. - Central recirculation zone contours. 

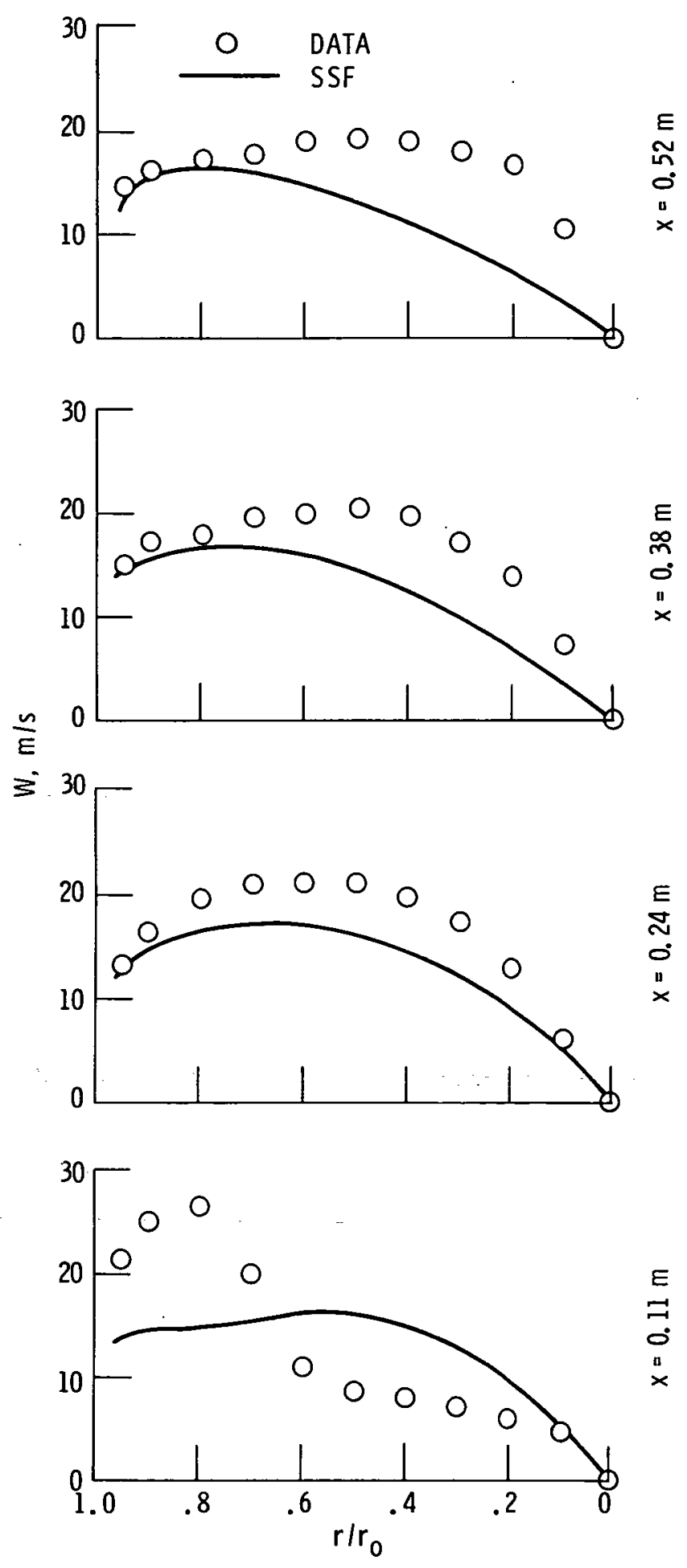

Figure 7. - Radial profiles of tangential velocity, $S=1.98$. 


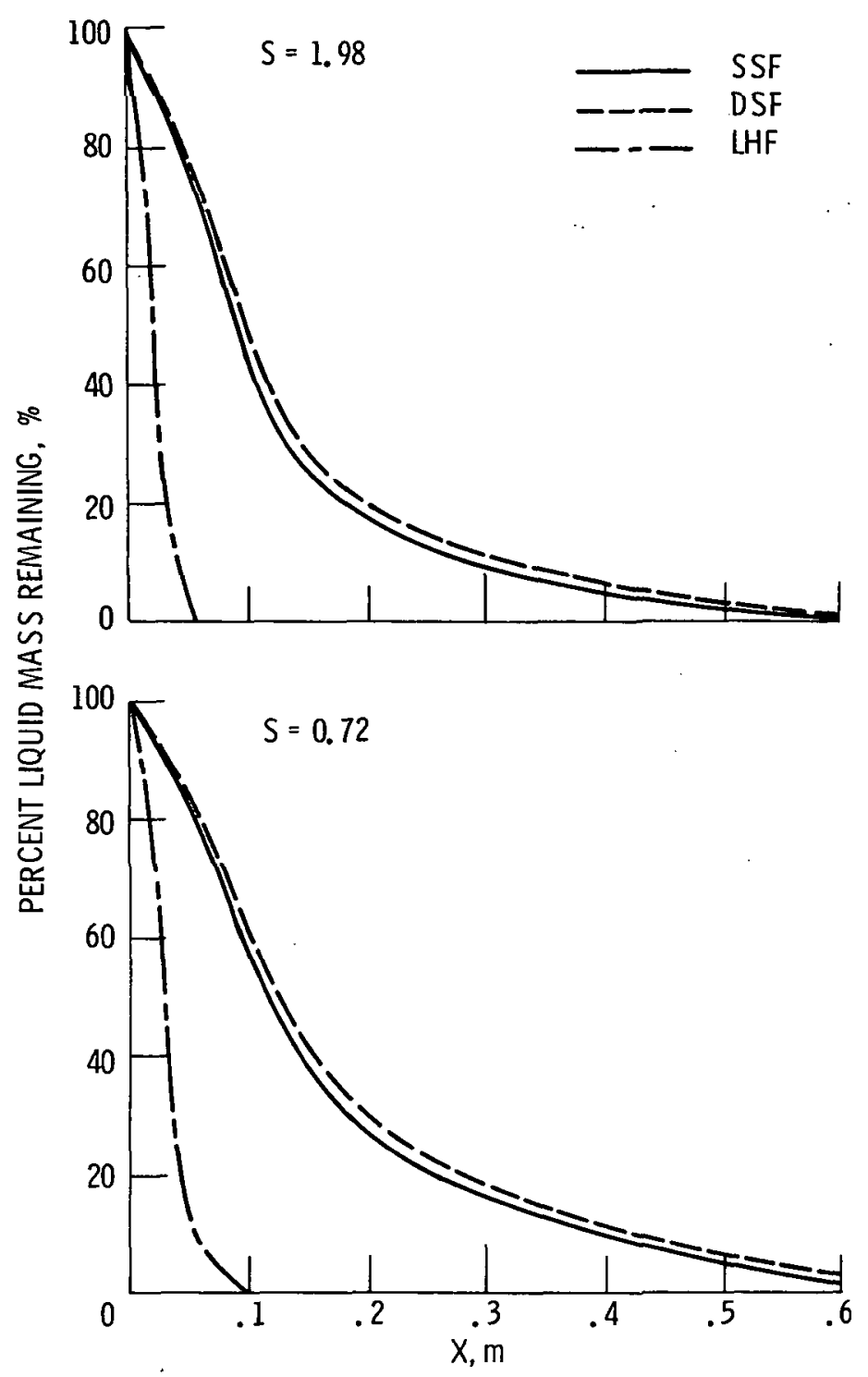

Figure 8. - Axial profiles of liquid mass flow rate. 


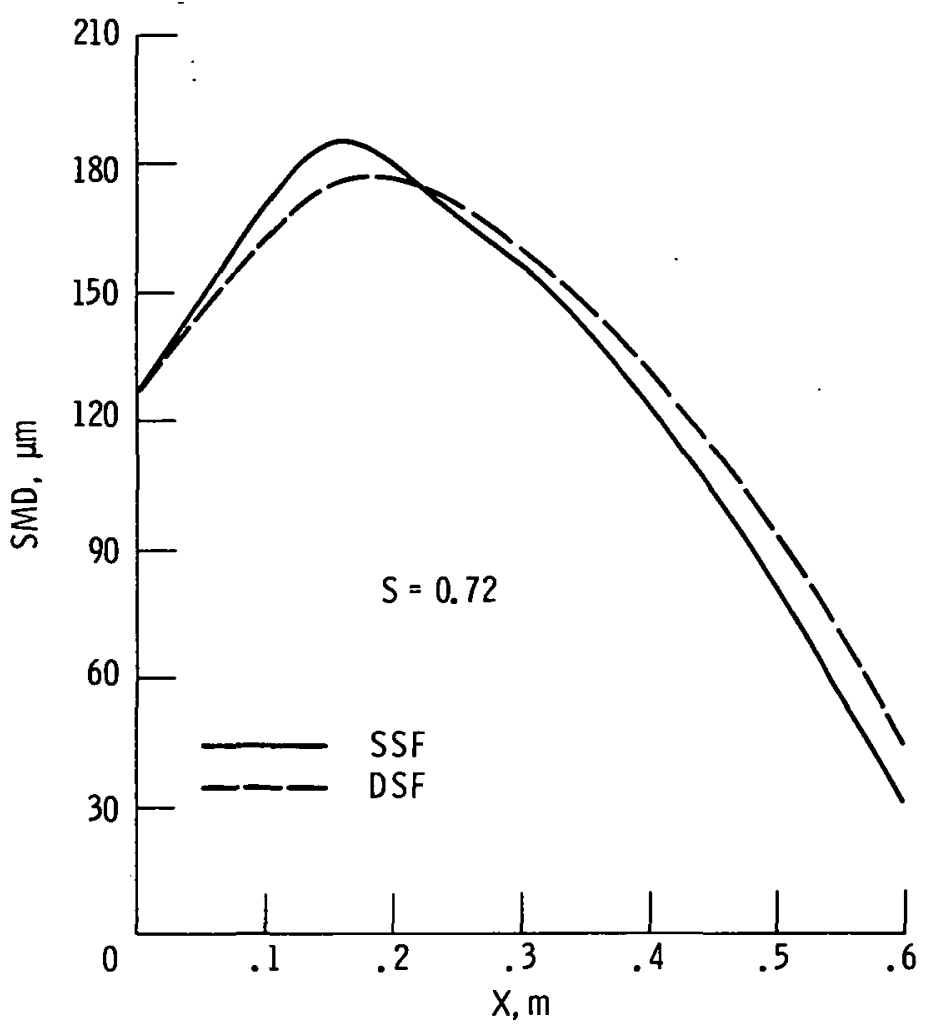

Figure 9. - Axial profiles of Sauter mean diameter. 


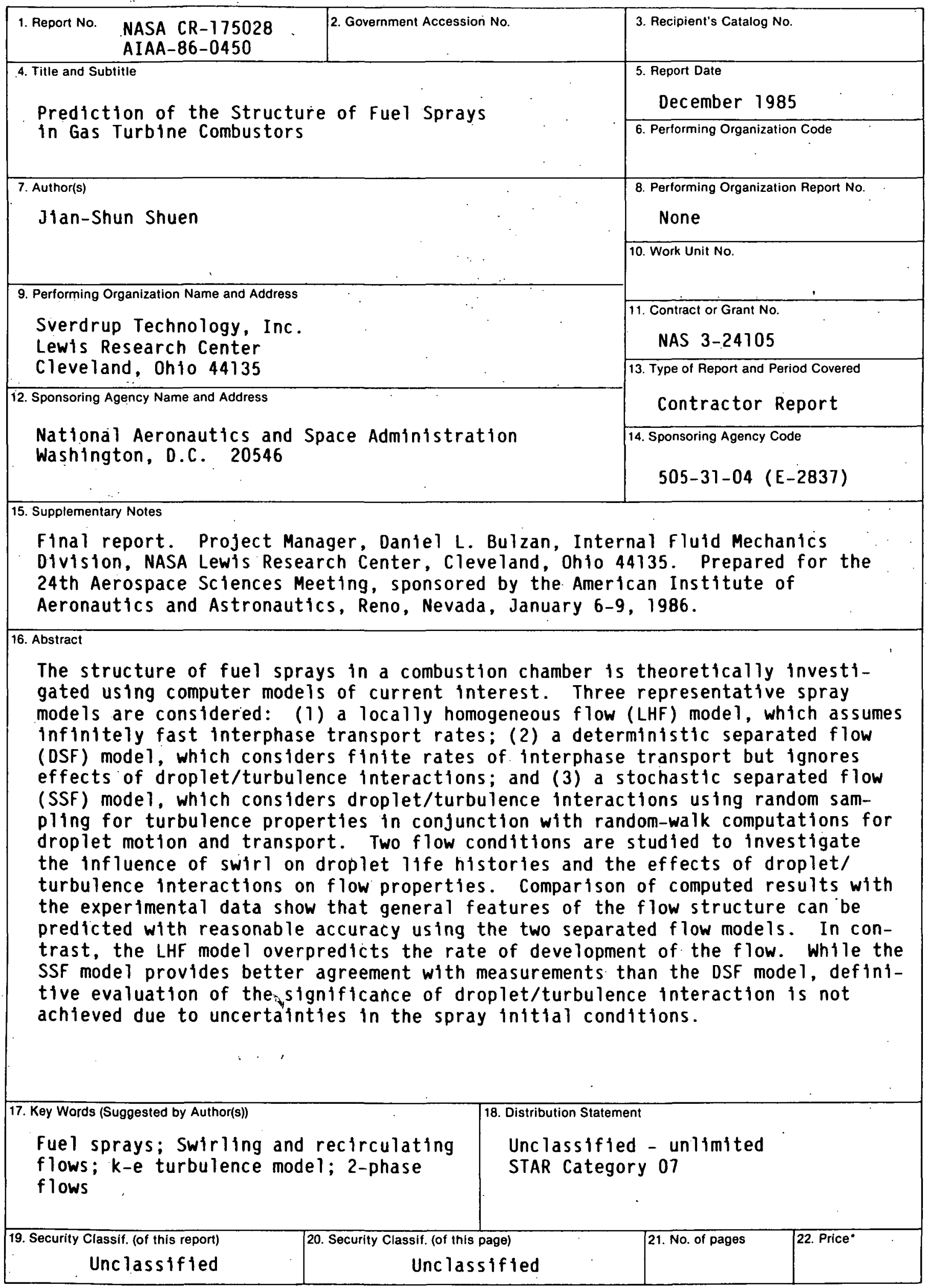

"For sale by the National Technical Information Service, Springfield, Virginia 22161. 
National Aeronautics and

Space Administration

Lewis Research Center

Cleveland. Ohio 44135

Official Business

Penalty for Private Use $\$ 300$
SECOND CLASS MAIL

ADDRESS CORRECTION REQUESTED

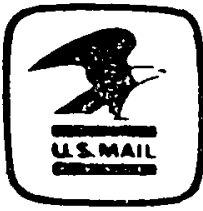

Postage and Fees Paid National Aeronautics and Space Administration NASA-451 